\title{
The Epidemiology and Economic Impact of Rhinosinusitis in Jos, North Central Nigeria
}

\author{
Adeyi A. Adoga** and Nuhu D. Ma'an²
}

${ }^{1}$ Consultant otolaryngologist, Otorhinolaryngology Unit, Department of Surgery, Jos University Teaching Hospital, PMB 2076, Jos, Plateau State, Nigeria ${ }^{2}$ Registrar, Otorhinolaryngology Unit, Department of Surgery, Jos University Teaching Hospital, PMB 2076, Jos, Plateau State, Nigeria

\begin{abstract}
Reports on the epidemiology of Rhinosinusitis in Nigeria are scarce and that on its economic impact is lacking. The aim of this work is to assess the prevalence and economic impact of rhinosinusitis in Jos, North-Central Nigeria.

This is a one year prospective survey of patients presenting with rhinosinusitis at the Jos University Teaching Hospital. Patients were evaluated for age, gender, occupation, and duration of illness, the economic cost of consultation, investigations, treatment and the type of complications seen.

Of the 486 new cases seen, $120(24.7 \%)$ patients aged between 3 and 60 years presented with rhinosinusitis. The male to female ratio was $1: 1.5$. Forty two (35\%) patients were aged between 31 and 40 years. Unemployed individuals constituted the majority. All patients were commenced on medical treatment and $16(1.3 \%)$ surgeries were done. Complication rate was $2.8 \%$. The recorded financial cost for initial treatment was 6,450 naira (\$42US) and 41,450 naira (\$269.2US) for patients requiring surgery/hospitalization.

The prevalence of rhinosinusitis is high in our center. The economic burden of this disease is enormous for patients in our region where the monthly minimum wage is 7,500 Naira (\$49US) and health care cost is the responsibility of the patient.
\end{abstract}

Keywords: Rhinosinusitis; Epidemiology; Economic impact; Jos; Nigeria

\section{Introduction}

Rhinosinusitis denotes the concurrent inflammatory and infectious process affecting the nasal cavities and the contiguous paranasal sinuses resulting from the interaction of multiple host and environmental factors [1].

It occurs worldwide and affects about $16 \%$ of the adult American population annually significantly impairing their quality of life [2]. The direct costs associated with rhinosinusitis are higher than the background costs due to increased clinic visits and prescribed medications and the indirect costs due to inactivity days leading to reduced productivity are enormous [3]. It has been found to be one of the most costly physical conditions for American citizens with approximately $\$ 6$ billion spent overall on its management [4].

The otorhinolaryngologist may see the more advanced cases of the disease but the primary care physician commonly attends to patients with features of the acute disease in which patients present with nasal obstruction, anterior and posterior nasal discharges, facial fullness with or without fever.

The diagnosis is clinical and radiological with high resolution computed tomography (CT) employing soft tissue windows showing areas of anatomical obstruction or discrete areas of diseased mucosa that are not seen by plain sinus $\mathrm{X}$-rays.

The clinician must bear in mind the fact that acute sinusitis can lead to orbital cellulitis and abscess, meningitis, intracranial abscesses, chronic maxillary dental irritation and abscess and laryngitis [5]. Prompt diagnosis and precise management is therefore required to avoid all these complications.

To the best of our knowledge some literatures from this part of the world have discussed the epidemiology of rhinosinusitis but nothing has been mentioned on the economic burden of this disease in our environment. Therefore, this work aims to highlight the epidemiology and economic impact of rhinosinusitis on a population in Jos, northcentral Nigeria.

\section{Materials and Methods}

This study was carried out at the otorhinolaryngology unit of the Jos University Teaching Hospital, Jos, North-central Nigeria.

After obtaining approval from the Ethical Committee of our institution, 120 patients presenting with rhinosinusitis from June 2007 to May 2008 were evaluated for age, gender, occupation, duration of illness, the economic cost of consultation, investigations, treatment and the type of complications seen.

There is a listed amount for each type of health care service rendered in our teaching hospital. These costs for consultation, investigations and treatment were used in our study to calculate the mean cost per patient per year. Patients were followed up throughout the study period and beyond for treatment outcome and possibility of development of complications.

\section{Results}

Of the 486 new patients seen in the study period, 120 patients aged

*Corresponding author: Dr. Adeyi A Adoga, Consultant otolaryngologist Otorhinolaryngology Unit, Department of Surgery, Jos University Teaching Hospital, PMB 2076, Jos, Plateau State, Nigeria, Tel: +234-803- 3897-283; E-mail: adeyiadoga@gmail.com

Received May 02, 2011; Accepted August 29, 2011; Published September 25 2011

Citation: Adoga AA, Ma'an ND (2011) The Epidemiology and Economic Impact of Rhinosinusitis in Jos, North Central Nigeria. J Clinic Res Bioeth 2:116. doi:10.4172/2155-9627.1000116

Copyright: (C) 2011 Adoga AA, et al. This is an open-access article distributed under the terms of the Creative Commons Attribution License, which permits unrestricted use, distribution, and reproduction in any medium, provided the original author and source are credited. 
Citation: Adoga AA, Ma'an ND (2011) The Epidemiology and Economic Impact of Rhinosinusitis in Jos, North Central Nigeria. J Clinic Res Bioeth 2:116. doi:10.4172/2155-9627.1000116

Page 2 of 3

between 3 and 60 years presented with rhinosinusitis accounting for $24.7 \%$ of the cases. There were $48(40 \%)$ males and 72 (60\%) females giving a Male: Female ratio of 1:1.5.

Forty two (35\%) of our patients were aged between 31 and 40 years (Table 1).

There were $26(21.7 \%)$ students, 30 (25\%) unskilled workers, 42 (35\%) unemployed individuals, 12 (10\%) professionals and $10(8.3 \%)$ patients whose occupation were not listed (Figure 1).

Sixty two percent of the patients seen were referred to our Otorhinolaryngology clinic by doctors in our hospital while $38 \%$ of referrals were from other hospitals in and outside Plateau state. Seventy percent of the referrals from within our hospital were by the General Medical Practitioners (GMP), 26\% were from Pediatricians and the rest from doctors of various other specialties.

The duration of symptoms before presentation ranged from 3 weeks to 5 months. The clinical features were nasal obstruction in 101 patients, unilateral or bilateral anterior rhinorrhea in 89 patients, posterior rhinorrhea in 76 patients and headache in 47 patients. Other features include facial fullness, dental pains, engorged inferior turbinates and mucoid and purulent nasal discharges on anterior rhinoscopy. The

\begin{tabular}{|ccccc|}
\hline Age & Females & Males & Total & Percentage \\
$0-10$ & 2 & 1 & 3 & 2.5 \\
$11-20$ & 9 & 5 & 14 & 11.7 \\
$21-30$ & 22 & 13 & 35 & 29.1 \\
$31-40$ & 27 & 15 & 42 & 35 \\
$41-50$ & 12 & 9 & 21 & 17.5 \\
$51-60$ & 5 & 0 & 5 & 4.2 \\
\hline
\end{tabular}

Table 1: Age distribution of patients with rhinosinusitis.

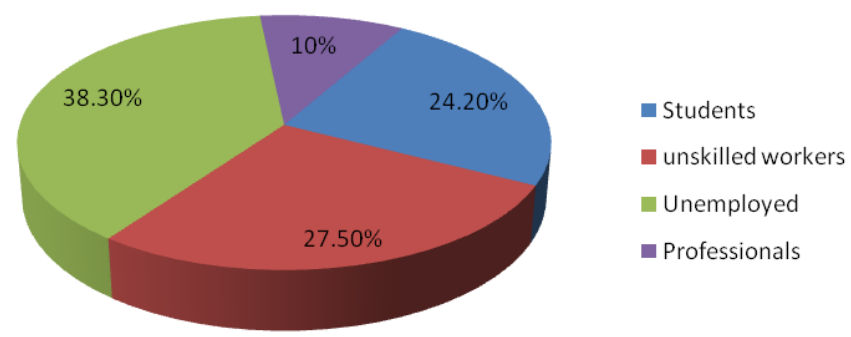

Figure 1: Category of patients seen with rhinosinusitis.

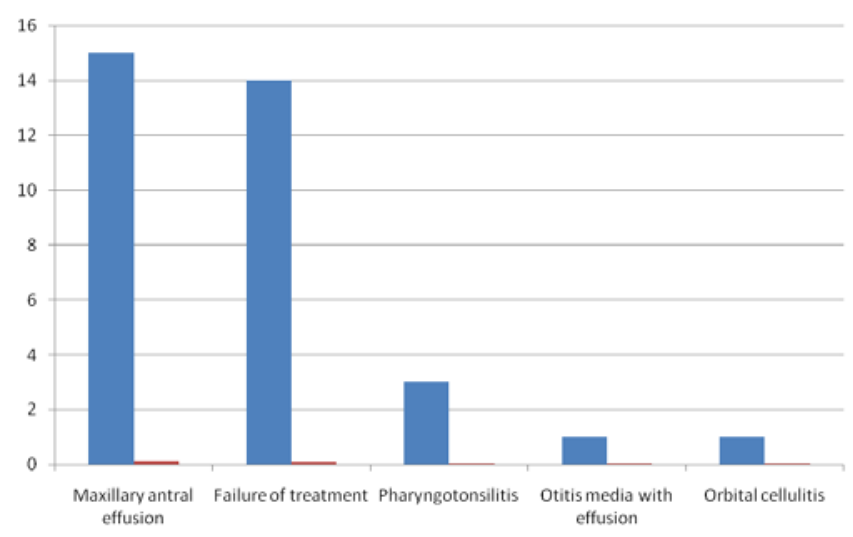

Figure 2: Complications of rhinosinusitis.

\begin{tabular}{|ll|}
\hline Service rendered & Cost of service (Naira) \\
\hline Registration/Initial consultation & 150 \\
Follow up visits(average of 10 visits/year) & 750 \\
Paranasal sinus X-ray & 2,750 \\
Medical treatment & 2,800 \\
Surgery/Hospitalization & 35,000 \\
\hline Total & $\mathbf{4 1 , 4 5 0}$ (\$269.2US) \\
\hline
\end{tabular}

Table 2: Cost of hospital visits, diagnosis and treatment per rhinosinusitis patient per year.

duration of missed work days for the employed workers ranged from 2 to 5 days and missed school days for students was 2 to 7 days.

Plain X-ray of the paranasal sinuses was done in all patients and computerized tomographic (CT) scan of the paranasal sinuses in 4 patients. Other investigations include full blood count, urea and electrolyte estimation and nasal smear cytology.

Medical treatment was by antibiotics, decongestants, antihistamines, steroid nasal sprays and steam inhalation. Surgery was carried out in some patients following failure of medical treatment and for those with the presence of complications. A total of $16(1.3 \%)$ surgeries were done in the study period and they were intranasal antrostomy $(n=12)$, intranasal antrostomy with polypectomy $(n=3)$, and Caldwel-luc procedure $(n=1)$. These surgeries were carried out on 11 unskilled workers, 4 unemployed patients and 1 professional.

The average number of clinic visits within the study period was 10 and a total of 502 follow up visits.

Comorbid conditions like nasal polyposis occurred in 7 (5.8\%) patients and allergy in $6(5 \%)$ patients.

The complications noted were maxillary antral effusion in 15 (12.5\%), failure of treatment in 14 (11.7\%) patients, pharyngotonsilitis in $3(2.5 \%)$ patients, otitis media with effusion in $1(0.8 \%)$ and orbital cellulitis in $1(0.8 \%)$ patients (Figure 2).

The recorded financial cost for initial treatment as shown on Table 2 was 6,450 naira (\$42US). For patients requiring surgery/ hospitalization, this figure increases to 41,450 naira (\$269.2US).

\section{Discussion}

Rhinosinusitis is a disease that is heterogeneous in its clinical and pathological manifestations, interfering with patients' quality of life and resulting in missed work and lost productivity therefore imposing a significant economic burden [6].

The wide range of clinical features of rhinosinusitis complicates the establishment of a working clinical diagnosis.

Patients with rhinosinusitis may present to a variety of practitioners including family care physicians, pediatricians, allergies, pulmonologists, neurosurgeons and otolaryngologists [7].

The reported prevalence of rhinosinusitis in patients available in literature is those from epidemiological studies conducted in other parts of the world. In our study, 486 patients were seen in the study period with $120(24.7 \%)$ patients presenting with rhinosinusitis. This is a high figure compared with the findings from other parts of the world in which prevalence rates of $1.95 \%$ to $16 \%$ have been recorded $[3,8,9]$.

Individuals in the fourth decades of life are more affected in our environment followed closely by those in their third decades. These represent the active work force in our society and with missed work days of between 2 and 5 days there is reduction in the productivity 
levels of these individuals at their various places of work. This has a negative impact overall on the economy. Rhinosinusitis causes indirect costs by reducing productivity at work and academic performance. It causes high rates of absenteeism and 'presenteeism' in which employees are present at work but underperforming [4].

Similar to findings in other series $[3,9,10]$, the prevalence of rhinosinusitis in our study was higher in females- $60 \%$ than in males- $40 \%$ and low income was associated with a higher prevalence of the disease in both sexes. Previous studies indicating increased susceptibility of females to asthma, chronic obstructive airway disease and rhinosinusitis may be the explanation for this [9].

The effects of rhinosinusitis on individual patients as well as society as a whole are extensive, resulting in significant direct and indirect costs as well as diminished quality of life. The direct costs result from the management of patients from increased clinic visits and the prescribed medications. The indirect costs are due to reduced activity days with missed work hours. This disease accounted for an average patient clinic visit of 10 within the one year study period and a total of 502 follow up visits.

The financial cost per patient for the initial hospital visit and treatment in our study was 6,450 naira (\$42.2US). For patients requiring surgery/hospitalization, this figure increases to 41,450 naira (\$271US). This is expensive considering that most of our patients are unemployed and even for those employed in a country where the minimum monthly wage is 7,500 naira (\$49US) this is a very significant burden on the patients because the cost of health care is usually the sole responsibility of the patient.

Proper and effective enforcement of the National Health Insurance Scheme (NHIS) in our environment can go a long way in helping especially the low and medium income earners to afford proper health care.

Rhinosinusitis produces remarkable socioeconomic burdens at all levels, national and international [4]. An analysis of a multiemployer database found rhinosinusitis to be very costly to employers [11]. A study conducted in 1996 in the United States (US) indicated that over 45 million people in the US are affected annually by this disease with an overall health care expenditure of $\$ 3.5$ billion [12]. This figure has risen to $\$ 6$ billion [13]. Conditions comorbid with rhinosinusitis occurred in a total of 13 patients. These conditions which are interrelated with rhinosinusitis in clinical manifestations have been found to exert significant strains on the economy. In 1996, allergic rhinitis was documented in up to $67 \%$ of patients with chronic rhinosinusitis, with expenditures for this disease totaling $\$ 3.5$ billion [12]. Plain paranasal sinus X-rays were ordered for all patients and the commonest radiological report was opacity of the maxillary sinus. $\mathrm{X}$-rays of the paranasal sinuses which give a high rate of false positive and negative results are inferior to computerized tomographic (CT) scans and magnetic resonance imaging (MRI) in making a diagnosis of rhinosinusitis which will give clear cut extents of the disease and assist the clinician in the proper treatment of patients. Only four patients in our series were able to afford CT scan. The high cost of this investigation35,000 naira ( $\$ 228.8 \mathrm{US}$ ) for a patient whose minimum monthly wage is 7,500 naira (\$49US) denies him/her adequate access to proper diagnosis and treatment. A complication rate of $2.8 \%$ was recorded in our series with maxillary antral effusion being the commonest complication. With the inability of patients to afford proper investigations like CT scans it is not surprising that we recorded a failure of treatment rate of $11.7 \%$. Endoscopic sinus surgery is a more effective and most recent mode of treatment of sinus diseases, rhinosinusitis inclusive [14] but we lack facilities for this mode of treatment in our center and many others around the country. It behooves government therefore to equip our hospitals with all the necessary facilities to provide even state of the art surgical care for the patients as it obtains in other parts of the world.

\section{Conclusion}

The prevalence of rhinosinusitis in our region is high. The economic burden of this disease is enormous for patients in our region where the monthly minimum wage is 7,500 Naira (\$49US) and health care cost is the responsibility of the patient. A systematic replication of this project on a national level would help in identifying the true epidemiology and economic impact of this disease. In addition, with time and money the diagnosis of rhinosinusitis will be more accurate using the nasal endoscope and the CT on a regular basis.

\section{References}

1. Kern RC, Conley DB, Walsh W, Chandra R, Kato A, et al. (2008) Perspectives on the etiology of chronic rhinosinusitis: an immune barrier hypothesis. Am J Rhinol 22: 549-559.

2. Gross, Charles W, Schlosser, Rodney J (2001) Prevalence of economic impact of rhinosinusitis. Current Opin Otolaryngol Head Neck Surg 9: 8-10.

3. Anand VK (2004) Epidemiology and economic impact of rhinosinusitis. Ann Otol Rhinol laryngol Suppl 193: 3-5.

4. Stewart M, Ferguson B, Fromer L (2010) Epidemiology and burden of nasa congestion. Int J Gen Med 3: 37-45.

5. Kutnick SL, Kerth JD (1976) Acute sinusitis and otitis: their complication and surgical treatment. Otolaryngol Clin North Am 9: 689-701.

6. Chan Y, Kuhn FA (2009) An update on the classifications, diagnosis and treatment of rhinosinusitis. Curr Opin Otolaryngol Head Neck Surg 17: 204-208.

7. Fokkeus W, Lund V, Mullol J (2007) European position paper on rhinosinusitis and nasal polyps group. Rhinol Suppl 20: 1-136.

8. Smith WM, Davidson TM, Murphy C (2009) Regional variations in chronic rhinosinusitis, 2003-2006. Otolaryngol Head Neck Surg 14: 347-52.

9. Chen Y, Dales R, Lin M (2003). The epidemiology of chronic rhinosinusitis in Canadians. Laryngoscope 113: 1199-1205.

10. da Lilly-Tariah OB (2006) Pattern of clinical features of chronic simple rhinosinusitis in Port-Harcourt. Niger J Clin Pract 9: 142-146.

11. Goetzel RZ, Hawkins K, Ozminkowski RJ, Wang S (2003) The health and productivity cost burden of the "top 10" physical and mental health conditions affecting six large US employers in 1999. J Occup Environ Med 45: 5-14.

12. Ray NF, Baraniuk JN, Thamer M, Rinehart CS, Gergen PJ, et al. (1999) Direct expenditures for the treatment of allergic rhinoconjuctivitis in 1996, including the contributions of related airway illnesses. J Allergy Clin Immunol (3pt1): 401 407.

13. Leggett JE (2004) Acute sinusitis. When-and when not-to prescribe antibiotics Postgrad Med 115: 13-19.

14. Poetker DM, Smith TL (2007) Adult chronic rhinosinusitis: surgical outcomes and the role of endoscopic sinus surgery. Curr Opin Otolaryngol Head Neck Surg 15: 6-9. 